\title{
Simple Resistance Exercise for 24 Weeks Decreases Alanine Ami- notransferase Levels in Patients with Non-Alcoholic Fatty Liver Disease
}

\section{(C) (2) (i) (:) Version 1}

Authors

Atsushi Takahashi ${ }^{1}$, Hiromichi Imaizumi ${ }^{1}$, Manabu Hayashi ${ }^{1}$, Ken Okai $^{1}$, Kazumichi Abe ${ }^{1}$, Keiji Usami $^{2}$, Nobuo Tanji ${ }^{3}$, Hiromasa Ohira ${ }^{1}$

Affiliations

1 Gastroenterology, Fukushima Medical University School of Medicine, Fukushima, Japan

2 Usami Medical Clinic, Internal Medicine, Koriyama, Japan

3 Gastroenterology, Watari Hospital, Fukushima, Japan

Key word

NAFLD, resistance exercise, ALT, 24 weeks

accepted after revision $\quad 31.08 .2016$

Bibliography

DOI http://dx.doi.org/10.1055/0042-117875

Published online: 2017 | Sports Medicine International Open

2017; 1: E2-E7

(c) Georg Thieme Verlag KG Stuttgart · New York

ISSN 2367-1890

Correspondence

Dr. Atsushi Takahashi, MD

Gastroenterology

Fukushima Medical University School of Medicine

1 Hikarigaoka, Fukushima

960-1295, Fukushima

Japan

Tel.: + 81/24/547 1202, Fax.: + 81/24/547 2005

junior@fmu.ac.jp

\begin{abstract}
Background: Exercise therapy is effective and recommended for non-alcoholic fatty liver disease (NAFLD) based on the efficacy of hepatic fat reduction. However, the efficacies of exercise therapy are based on shortterm intervention. Moreover, no reports have examined whether significant reductions in serum levels of alanine aminotransferase (ALT) are achieved with exercise therapy in patients with NAFLD. The aim of this study is to assess the effects of simple resistance exercise for 24 weeks in NAFLD. Methods: 59 patients with NAFLD were assigned to a resistance exercise group $(n=28)$ or a control group $(n=31)$. The resistance exercise group performed 2 exercises (push-ups and squats) 3 times a week on nonconsecutive days for a trial periods of 24 weeks. Patients in the control group proceeded with regular physical activities under a restricted diet throughout the study. The effects of exercise were compared between groups after 24 weeks.

Results: Mean ALT level, homeostasis model assessment-estimated insulin resistance index and hepatic steatosis grade were all decreased in the resistance exercise group. Changes in ALT levels correlated negatively with changes in muscle:body weight ratio in the exercise group. Conclusion: These data demonstrate that 24 weeks of simple resistance exercise comprising squats and push-ups represents an effective treatment for NAFLD.
\end{abstract}

\section{Introduction}

Exercise is an important treatment for both non-alcoholic fatty liver disease (NAFLD) and metabolism-associated diseases. Exercise therapies are generally classified as aerobic or resistance type, and both are recommended as standard therapies for diabetes mellitus [22]. The effects of aerobic exercise have been established based on many studies $[12,13,23]$, so only aerobic exercises are recommended in the Japanese clinical guidelines for NAFLD and non-alcoholic steatohepatitis (NASH) [22]. However, some recent studies have reported the efficacy of resistance exercise in patients with $\operatorname{NAFLD}[2,9,24]$.

Resistance exercise can comprise many methods to train muscles and generally needs training instruments. In fact, at least 8 different types of training methods have been applied to patients with NAFLD $[2,9,24]$. We recently reported that a simple non-instrumental resistance exercise, using body weight as a load, helps to improve the characteristics of metabolic syndrome in patients with NAFLD [19]. Moreover, compliance with the simple resistance exercise program did not significantly correlate with patient background characteristics such as age, sex, body mass index (BMI) and metabolic complications. These facts imply that a simple resistance exercise program might offer advantages over other types of resistance exercise using training instruments in maintaining exercise adherence.

Plasma alanine aminotransferase (ALT) levels are often used as a screening marker for NAFLD. Some studies have shown that the presence of diabetes or insulin resistance is associated with higher ALT levels $[3,8,15]$. Moreover, Maximos et al. recently revealed that liver fat and adipose tissue insulin resistance are major factors associated with elevated levels of plasma aminotransferases in NAFLD [14]. Although resistance exercise improves metabolic parameters, including liver adiposity, improvement of liver enzymes through 
resistance exercise has not yet been confirmed, including in our own report $[2,9,19,24]$. The short-term nature of continued resistance exercise may be one reason for this lack of change, as previous studies have involved relatively short periods of exercise of about 8-16 weeks. We therefore evaluated the effects of push-ups and squats continued for 24 weeks in patients with NAFLD.

\section{Methods \\ Participants}

The diagnosis of NAFLD was based on the Asia-Pacific Working Party guidelines for NAFLD [6]. Exclusion criteria comprised: evidence of other liver diseases such as chronic hepatitis $C$, chronic hepatitis $B$, autoimmune hepatitis, primary biliary cirrhosis or alcoholic liver disease ( $>20 \mathrm{~g}$ of alcohol/day); consumption of weight-loss agents, corticosteroids, tamoxifen, herbal medicines or anti-diabetic agents (patients with metabolic complications who were stable for > 6 months without additional drugs included); or heart or kidney disease or other preexisting medical conditions that might prevent participation in the exercise program. Among the 69 patients who agreed to participate in this study, 10 were excluded due to protocol violations or missing data or loss to follow-up. The remaining 59 patients were assigned to a group that performed resistance exercise or a control group. All 59 patients had a sedentary lifestyle before the study intervention period. The flowchart for patient selection is shown in $>$ Fig. 1. In the present study, controls added to the cohort that we previously reported [19].

All protocols were approved by the ethics committees of Watari
Hospital and Fukushima Medical University School of Medicine (Fukushima, Japan). All patients provided written informed consent to participate prior to enrollment in the study, which conformed to the ethical standards of sports and exercise science research [10].

\section{Procedures}

\section{Resistance exercise group}

Patients in the exercise group were educated about resistance exercise comprising push-ups and squats at the beginning of the study. They then performed 3 sets of 10 push-ups and 3 sets of 10 squats with a 1-min interval between each set over a period of 20-30 min, and recorded their compliance with the regimen. Detailed methods have been shown in our previous report [19].

\section{Controls}

The control group was educated about dietary restrictions and encouraged to participate in regular physical activities in accordance with the American Gastroenterological Association for NAFLD [1] and the Physical Activity of Health Promotion guidelines recommended by the Ministry of Health, Labor and Welfare of Japan [16].

\section{Outcome measures}

Total body weight and composition were measured using a DC-320 bioelectrical impedance analyzer (Tanita Corporation, Tokyo, Japan). BMI was calculated by dividing body weight in kilograms by the square of the height in meters without using a bioelectrical im-

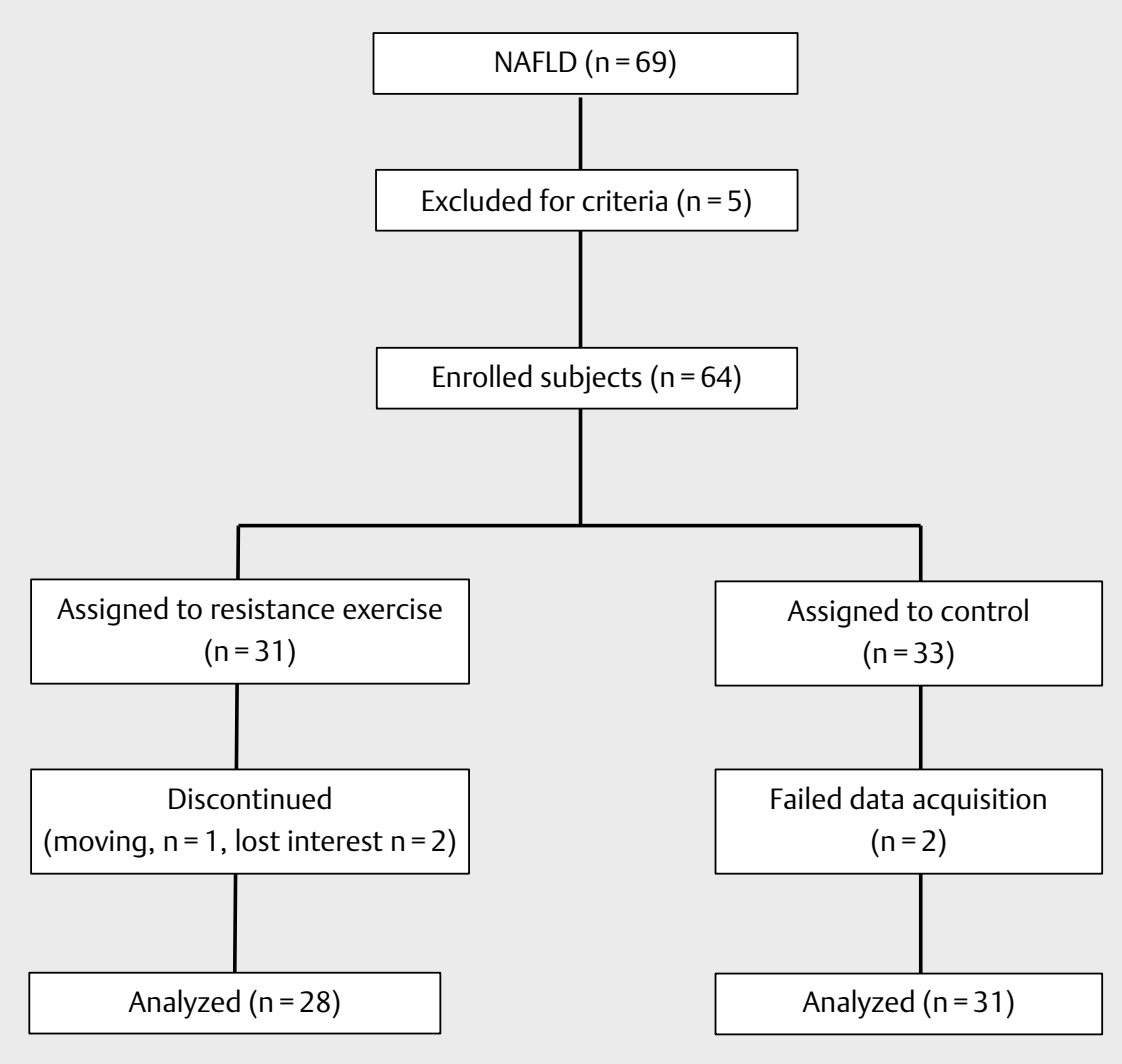

- Fig. 1 Participant flow diagram in the present study. NAFLD, non-alcoholic fatty liver disease 
pedance analyzer. Venous blood samples were collected in the morning after an overnight fast. Levels of aspartate aminotransferase (AST), ALT, y-glutamyl transpeptidase, high-density lipoprotein cholesterol, low-density lipoprotein cholesterol, triglyceride, fasting plasma glucose (FPG) and plasma insulin were determined using standard methods. Insulin resistance was calculated according to the homeostasis model assessment-estimated insulin resistance (HOMA-IR) index using the following formula: $\mathrm{HOMA}-\mathrm{IR}=\mathrm{FPG}$ $(\mathrm{mg} / \mathrm{dL}) \times$ fasting plasma insulin $(\mu \mathrm{U} / \mathrm{mL}) / 405$.

Hepatic steatosis was assessed according to the methods of Saadeh et al. using LogiQ 7 software (GE Health Care, Piscataway, NJ) and ultrasonography (US) on a ProSound Alpha 7 system (Hitachi-Aloka Medical, Tokyo, Japan) [18], then graded according to the severity of echogenicity. Grade 1 included a slight, diffuse increase in fine echoes in liver parenchyma, with normal visualization of the diaphragm and intrahepatic vessel borders; Grade 2 showed a moderate, diffuse increase in fine echoes with slightly impaired visualization of intrahepatic vessels and diaphragm; and Grade 3 depicted an obvious increase in fine echoes with poor or obscured visualization of the intrahepatic vessel borders, diaphragm and posterior right liver lobe.

\section{Statistical analyses}

Results are expressed as mean \pm standard deviation. Differences between groups were analyzed using the Mann-Whitney U test. After testing for a normal distribution, significant within-group differences between before and after 24 weeks were determined using paired Student's t-tests. Treatment group $\times$ time interactions were assessed using 2-way (group $\times$ time) repeated-measures ANOVA. Dichotomous variables were compared using the $x^{2}$ test. Correlations between variables were measured using Pearson's correlation coefficient or Spearman's rank correlations when variables were not normally distributed. Values of $\mathrm{P}<0.05$ were considered indicative of significant differences.

\section{Results}

Between January 2012 and September 2014, 59 patients (female, $n=42$ ) with NAFLD were assigned to receive either 24 weeks of resistance exercise $(n=28)$ or lifestyle counseling ( $n=31$; controls). - Table 1 summarizes the clinical characteristics of the patients. The 2 groups were almost matched for baseline characteristics, with the exception of ALP and hemoglobin A1c levels and prevalence of hypertension ( $\triangleright$ Table 1 ). No patients in the exercise group developed severe adverse events during the study period. Compliance with the resistance exercise program was $71.7 \%$, and did not correlate significantly with patient background characteristics such as age, sex, BMI or metabolic complications.

A significant time-by-treatment interaction was evident between body weight $(p=0.013)$ and BMI $(p<0.001)$. Moreover, a significant time-by-treatment interaction was also seen between ALT levels and HOMA-IR. ALT levels (76.9 \pm 63.4 vs. $59.3 \pm 49.2$, $\mathrm{p}=0.003)$ and HOMA-IR $(4.0 \pm 2.9$ vs. $3.1 \pm 1.6, \mathrm{p}=0.025)$ were significantly decreased with exercise, but not in the control group. We also found a significant time-by-treatment interaction in hepatic steatosis grade $(p=0.021)$. Moreover, hepatic steatosis grade $(2.00 \pm 0.82$ vs. $1.55 \pm 0.71, p=0.001)$ was significantly decreased with exercise, but not in the control group ( $\triangleright$ Table 2 ).

- Table 1 Baseline characteristics of study subjects.

\begin{tabular}{|c|c|c|c|}
\hline Characteristic & Control $(n=31)$ & Resistance exercise $(n=28)$ & $\boldsymbol{P}$ \\
\hline Sex (male/female) & $9 / 22$ & $8 / 20$ & 0.969 \\
\hline Age (year) & $52.9 \pm 15.4$ & $56.7 \pm 12.0$ & 0.362 \\
\hline Body weight (kg) & $72.0 \pm 14.4$ & $70.7 \pm 11.7$ & 0.785 \\
\hline BMI $\left(\mathrm{kg} / \mathrm{m}^{2}\right)$ & $28.4 \pm 3.9$ & $28.3 \pm 3.2$ & 0.834 \\
\hline AST (IU/L) & $44.1 \pm 21.7$ & $46.5 \pm 25.7$ & 0.710 \\
\hline ALT (IU/L) & $67.4 \pm 39.3$ & $76.9 \pm 63.4$ & 0.982 \\
\hline $\operatorname{ALP}(I U / L)$ & $223.8 \pm 78.9$ & $261.4 \pm 94.4$ & 0.025 \\
\hline Y-GTP (IU/L) & $51.6 \pm 24.7$ & $51.3 \pm 33.5$ & 0.7 \\
\hline LDL-C (mg/dL) & $129.3 \pm 43.1$ & $127.5 \pm 29.2$ & 0.933 \\
\hline $\mathrm{HDL}-\mathrm{C}(\mathrm{mg} / \mathrm{dL})$ & $54.0 \pm 15.2$ & $54.8 \pm 17.4$ & 0.482 \\
\hline Triglycerides (mg/dL) & $165.1 \pm 97.8$ & $143.3 \pm 86.8$ & 0.527 \\
\hline Ferritin (ng/dL) & $160.5 \pm 170.7$ & $213.0 \pm 207.8$ & 0.191 \\
\hline Blood glucose (mg/dL) & $107.7 \pm 20.4$ & $113.8 \pm 16.4$ & 0.082 \\
\hline Insulin $(\mu \mathrm{U} / \mathrm{mL})$ & $19.3 \pm 22.1$ & $13.6 \pm 8.4$ & 0.513 \\
\hline HOMA-IR & $5.6 \pm 8.2$ & $4.0 \pm 2.9$ & 0.846 \\
\hline HbA1c (\%) & $5.8 \pm 0.7$ & $6.1 \pm 0.6$ & $0.024^{*}$ \\
\hline Hepatic steatosis (score) & $2.34 \pm 0.82$ & $2.00 \pm 0.82$ & 0.090 \\
\hline Diabetes mellitus (cases) & 5 & 9 & 0.191 \\
\hline Dyslipidemia (cases) & 23 & 17 & 0.269 \\
\hline Hypertension (cases) & 5 & 14 & $0.005^{*}$ \\
\hline
\end{tabular}


- Table 2 Changes in physical and clinical parameters after 24 weeks of resistance exercise.

\begin{tabular}{|c|c|c|c|c|c|c|c|}
\hline & \multirow{2}{*}{$\begin{array}{l}\text { Control } \\
\text { Baseline } \\
\end{array}$} & \multirow{2}{*}{$\begin{array}{c}\begin{array}{c}\text { Resistance } \\
\text { exercise }\end{array} \\
24 \text { weeks }\end{array}$} & \multicolumn{5}{|c|}{ Time $\times$ treatment interaction $(p)$} \\
\hline & & & $p$ & Baseline & 24 weeks & $p$ & \\
\hline Body weight (kg) & $72.0 \pm 14.4$ & $72.7 \pm 14.6$ & $0.044^{*}$ & $70.7 \pm 11.7$ & $70.1 \pm 11.2$ & 0.125 & $0.013 \dagger$ \\
\hline BMI $\left(\mathrm{kg} / \mathrm{m}^{2}\right)$ & $28.4 \pm 3.9$ & $28.7 \pm 3.8$ & $0.040^{*}$ & $28.3 \pm 3.2$ & $28.0 \pm 3.1$ & 0.058 & $<0.001 \dagger$ \\
\hline Body fat $(\mathrm{kg})$ & $26.7 \pm 7.2$ & $27.1 \pm 7.4$ & 0.304 & $26.4 \pm 6.2$ & $26.3 \pm 6.8$ & 0.892 & 0.486 \\
\hline Muscle $(\mathrm{kg})$ & $43.5 \pm 10.8$ & $43.7 \pm 11.0$ & 0.649 & $41.9 \pm 9.5$ & $42.1 \pm 9.2$ & 0.375 & 0.848 \\
\hline Muscle/Body weight (\%) & $59.4 \pm 6.8$ & $59.3 \pm 7.2$ & 0.715 & $59.1 \pm 7.1$ & $59.9 \pm 6.8$ & 0.022 & 0.077 \\
\hline AST (IU/L) & $44.1 \pm 21.7$ & $45.3 \pm 18.5$ & 0.778 & $46.5 \pm 25.7$ & $40.0 \pm 20.6$ & 0.048 & 0.165 \\
\hline $\operatorname{ALT}(I U / L)$ & $67.4 \pm 39.3$ & $74.2 \pm 44.0$ & 0.241 & $76.9 \pm 63.4$ & $59.3 \pm 49.2$ & $0.003^{*}$ & $0.004 \dagger$ \\
\hline $\operatorname{ALP}(\mathrm{IU} / \mathrm{L})$ & $230.1 \pm 69.2$ & $240.9 \pm 70.3$ & 0.168 & $261.4 \pm 94.4$ & $264.7 \pm 79.3$ & 0.844 & 0.659 \\
\hline $\mathrm{Y}$-GTP (IU/L) & $51.7 \pm 25.1$ & $56.1 \pm 27.1$ & 0.103 & $51.2 \pm 32.8$ & $48.8 \pm 33.4$ & 0.250 & 0.100 \\
\hline $\mathrm{LDL}-\mathrm{C}(\mathrm{mg} / \mathrm{dL})$ & $132.4 \pm 40.5$ & $136.2 \pm 47.5$ & 0.316 & $127.5 \pm 29.2$ & $124.0 \pm 30.9$ & 0.376 & 0.180 \\
\hline $\mathrm{HDL}-\mathrm{C}(\mathrm{mg} / \mathrm{dL})$ & $54.0 \pm 15.2$ & $52.7 \pm 13.5$ & 0.469 & $54.8 \pm 17.4$ & $52.4 \pm 14.2$ & 0.108 & 0.644 \\
\hline Triglycerides (mg/dL) & $143.3 \pm 86.8$ & $151.4 \pm 92.3$ & 0.557 & $162.9 \pm 96.8$ & $171.1 \pm 113.4$ & 0.479 & 0.994 \\
\hline Ferritin (ng/dL) & $160.5 \pm 170.7$ & $156.1 \pm 120.1$ & 0.836 & $191.5 \pm 174.7$ & $150.1 \pm 117.5$ & 0.013 & 0.175 \\
\hline Blood glucose (mg/dL) & $105.3 \pm 16.2$ & $106.2 \pm 14.4$ & 0.938 & $113.8 \pm 16.4$ & $109.4 \pm 12.8$ & 0.045 & 0.096 \\
\hline Insulin $(\mu \mathrm{U} / \mathrm{mL})$ & $13.8 \pm 7.7$ & $17.5 \pm 17.4$ & 0.449 & $13.6 \pm 8.4$ & $11.1 \pm 4.6$ & 0.040 & 0.084 \\
\hline HOMA-IR & $3.6 \pm 2.1$ & $3.9 \pm 2.5$ & 0.407 & $4.0 \pm 2.9$ & $3.1 \pm 1.6$ & $0.025^{*}$ & $0.020 \dagger$ \\
\hline HbA1c (\%) & $5.8 \pm 0.7$ & $5.9 \pm 0.6$ & 0.579 & $6.0 \pm 0.6$ & $6.0 \pm 0.7$ & 0.261 & 0.205 \\
\hline Hepatic steatosis & $2.40 \pm 0.81$ & $2.29 \pm 0.77$ & 0.184 & $2.00 \pm 0.82$ & $1.55 \pm 0.71$ & $0.001^{*}$ & $0.021 \dagger$ \\
\hline
\end{tabular}

Values are expressed as means \pm SD. Severity of hepatic steatosis graded according to criteria of Saadeh et al. [17]. Significant within-group differences between before and after 24 weeks were determined using paired Student's $t$-tests. Treatment group $\times$ time interaction were assessed using 2 -way (group $\times$ time) repeated measures ANOVA. * Significant differences between baseline and after 24 weeks $(p<0.05)$. $\dagger$ Significant differences time $\times$ treatment interaction $(p<0.05)$. BMI, body mass index; AST, aspartate aminotransferase; ALT, alanine aminotransferase; ALP, alkaline phosphatase; Y-GTP, gamma-glutamyl transpeptidase; LDL-C, low-density lipoprotein cholesterol; HDL-C, high-density lipoprotein cholesterol; HOMA-IR, homeostasis model assessment of insulin resistance

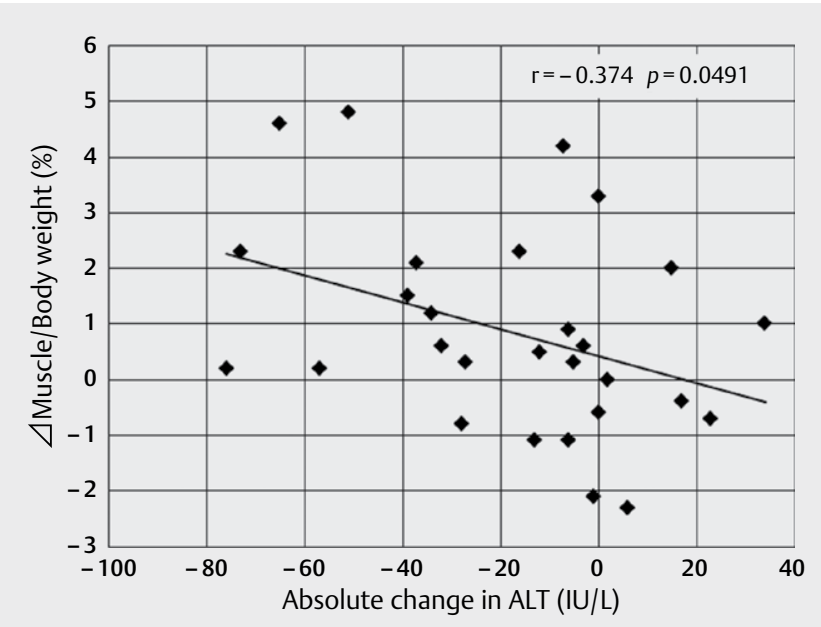

Fig. 2 Association between change in muscle:body weight ratio and absolute change in ALT after 24 weeks of resistance exercise. $\Delta=$ variable after exercise - variable before exercise. Pearson's correlation coefficient $(r)$ was used to determine correlations.

Changes in ALT levels $(r=-0.374, p=0.049)$ correlated negatively with changes in the muscle:body weight ratio in the exercise group ( $\triangleright$ Fig. 2$)$. Changes in HOMA-IR $(r=-0.362, p=0.060)$ showed a weak, negative correlation with changes in the muscle:body weight ratio. Moreover, changes in ALT levels $(r=0.375$, $\mathrm{p}=0.052$ ) displayed a weak, positive correlation with changes in hepatic steatosis grade. No significant association was seen between changes in ALT and HOMA-IR $(r=0.148, p=0.443)$ in the overall exercise group, but a positive correlation was identified $(r=0.556, p=0.013)$ in the exercise group without diabetes mellitus $(n=19)$.

\section{Discussion}

This is the first study to find that simple resistance exercise achieved significant reductions in ALT levels after 24 weeks. This was accompanied by decreases in HOMA-IR and liver adiposity. Exercise has been established as a representative lifestyle therapy in NAFLD based largely on aerobic exercise, but the efficacy of resistance exercise in NAFLD has recently been reported [2, 9, 19, 24]. Hallsworth et al. first reported that resistance exercise improved NAFLD through reductions in liver lipid levels and improved insulin sensitivity [9]. Moreover, Bacchi et al. revealed resistance exercise as useful along with aerobic exercise in NAFLD with type 2 diabetes [2]. Resistance exercise offers advantages over aerobic exercise in terms of the low cardiorespiratory demand. On the other hand, specialized equipment and specific exercise methods might make resistance exercise less accessible than aerobic exercise. However, we recently reported that simple resistance exercises comprising 
squats and push-ups helped to improve the characteristics of metabolic syndrome in patients with NAFLD in the same way as traditional resistance exercises using equipment [19].

Physicians often pay attention to the risk of NASH or NAFLD indicated by the risk of elevated plasma ALT levels. In fact, the use of serum ALT levels is recommended in the initial screening for NAFLD in obese youth by current guidelines [5]. Maximos et al. recently revealed that the elevation of plasma ALT is mainly due to adipose tissue insulin resistance and liver triglyceride content [14]. Improving ALT is therefore one of the therapeutic targets in NAFLD. Ueno et al. first demonstrated improvement of serum aminotransferase levels and liver histology in NAFLD using a combination of aerobic exercise and dietary restrictions [21]. Although little research has demonstrated reductions in liver fat in NAFLD due to aerobic exercise per se [12], few reports have demonstrated significant changes in ALT levels after intervention using aerobic exercise. Thompson et al. reported that 6 months of aerobic exercise significantly decreased serum levels of ALT in middle-aged men [20], but no other reports have shown significant ALT reductions on resistance exercise. This study is thus the first to mention significant reductions achieved through resistance training. The major reason for the significant reduction in ALT was probably the relatively long duration of exercise intervention. Previous studies have largely been short-term interventions, not only for aerobic exercise but also for resistance exercise. We likewise could not find any significant reduction in ALT after 12 weeks of intervention using the same resistance exercise. In fact, Thomson et al. confirmed that ALT took longer to change and showed significant reductions after 6 months of aerobic exercise intervention, but not with 3 months of intervention [20]. Moreover, exercise intensity and baseline ALT levels might be associated with the lack of significant changes in ALT levels. In the present study, baseline serum ALT levels were higher than those in previous reports.

Contracting skeletal muscle stimulates muscle glucose uptake by glucose transporter type 4 (GLUT4) in addition to insulin [11]. Resistance exercise upregulates GLUT 4 expression in skeletal muscle, thus leading to a whole-body increase in glucose metabolism [7]. The present study confirmed a weak negative correlation between changes in HOMA-IR and muscle:body weight ratio. Moreover, a significant negative correlation was seen between changes in ALT and muscle:body weight ratio in the present study. The increase in muscle:body weight ratio was one reason for the efficacy of resistance training in the present study. Skeletal muscle has also recently been attracting attention as an endocrine organ that produces and releases myokines [17]. Myokines such as irisin and insulin-like growth factor 1 play roles in glycolipid metabolism, and may thus have contributed to the improvements in NAFLD in the present study.

The efficacy of exercise therapy depends on the sustainability or maintenance of exercise. Squats and push-ups are simple resistance exercises requiring no specific equipment and compliance does not correlate with patient background characteristics [19]. Our exercise program (push-ups and squats) could thus have contributed to the good results in the present study through the relatively long-term sustainability of exercise.
This study has some limitations. We did not monitor physical activity and food intake. Therefore, the differences in food intake might affect the results. Moreover, the efficacy of exercise therapy per se is often argued in effects of dietary modification. However, we confirmed a significant increase in muscle:body weight ratio in the exercise group, reflecting the effects of resistance exercise. Moreover, changes in muscle:body weight ratio correlated negatively with changes in ALT levels. This reflects improvement of glycolipid metabolism by a resistance exercise-induced decrease of ALT levels and liver triglyceride content. In addition, a direct benefit of exercise therapy often provides a practical intervention target beyond weight loss [12]. There was also no significant change in body weight seen in the exercise group in the present study. These findings are consistent with our results reflecting the efficacy of exercise therapy per se.

Another limitation was that liver adiposity was analyzed on US, as the most popular and minimally invasive method of detecting fatty liver disease. Although Saadeh et al. [18] and Celle et al. [4] independently reported positive associations between fat content as determined by liver histology and US, this latter modality involves several issues such as technical difficulties in scanning obese patients and the subjective judgment of the operator [18]. However, we confirmed a weak positive correlation between changes in ALT levels and the grade of hepatic steatosis. This finding was presumably associated with the fact that plasma ALT elevations reflect increasing liver fat content [14]. Evaluation of liver fat using US in the present study was thus justifiable. Third, the sample size was small and not randomized. In the future, a randomized, larger-scale, longer-term study is required to confirm the value of simple resistance exercise as a treatment for NAFLD.

In conclusion, the results of this trial demonstrate for the first time that 24 weeks of simple resistance comprising squats and push-ups are effective in reducing ALT in patients with NAFLD. Our data indicate that this program can provide benefits for the management of NAFLD.

\section{Acknowledgements}

This study was supported by a fellowship from Fukushima Medical University.

\section{Conflict of interest}

The authors declare that they have no conflict of interest.

\section{References}

[1] American Gastroenterological Association. American Gastroenterological Association medical position statement: nonalcoholic fatty liver disease. Gastroenterology 2002; 123: 1702-1704

[2] Bacchi E, Negri C, Targher G, Faccioli N, Lanza M, Zoppini G, Zanolin E, Schena F, Bonora E, Moghetti P. Both resistance training and aerobic training reduce hepatic fat content in type 2 diabetic subjects with NAFLD (The RAED2 randomized trial). Hepatology 2013; 58: 1287-1295 
[3] Burgert TS, Taksali SE, Dziura J, Goodman TR, Yeckel CW, Papademetris X, Constable RT, Weiss R, Tamborlane WV, Savoye M, Seyal AA, Caprio $S$. Alanine aminotransferase levels and fatty liver in childhood obesity: associations with insulin resistance, adiponectin, and visceral fat. J Clin Endocrinol Metab 2006; 91: 4287-4294

[4] Celle G, Savarino V, Picciotto A, Magnolia MR, Scalabrini P, Dodero M. Is hepatic ultrasonography a valid alternative tool to liver biopsy? Report on 507 cases studied with both techniques. Dig Dis Sci 1988; 33: $467-471$

[5] Chalasani N, Younossi Z, Lavine JE et al. The diagnosis and management of non-alcoholic fatty liver disease: practice guideline by the American Association for the Study of Liver Diseases, American College of Gastroenterology, and the American Gastroenterological Association. Hepatology 2012; 55: 2005-2023

[6] Farrell GC, Chitturi S, Lau GK, Diehl AM, Brunt EM, Cusi K, Charlton M, Sanyal AJ. Asia-Pacific Working Party on NAFLD. Guidelines for the assessment and management of non-alcoholic fatty liver disease in the Asia-Pacific region: executive summary. J Gastroenterol Hepatol 2007; 22: 775-777

[7] Ferrara CM, Goldberg AP, Ortmeyer HK, Ryan AS. Effects of aerobic and resistive exercise training on glucose disposal and skeletal muscle metabolism in older men. J Gerontol A Biol Sci Med Sci 2006; 61: 480-487

[8] Fracanzani AL, Valenti L, Bugianesi E, Andreoletti M, Colli A, Vanni E, Bertelli C, Fatta E, Bignamini D, Marchesini G, Fargion S. Risk of severe liver disease in nonalcoholic fatty liver disease with normal aminotransferase levels: a role for insulin resistance and diabetes. Hepatology 2008; 48: 792-798

[9] Hallsworth K, Fattakhova G, Hollingsworth KG, Thoma C, Moore S, Taylor R, Day CP, Trenell MI. Resistance exercise reduces liver fat and its mediators in non-alcoholic fatty liver disease independent of weight loss. Gut 2011; 60: 1278-1283

[10] Harriss D], Atkinson G. Ethical standards in sport and exercise science research: 2016 update. Int J Sports Med 2015; 36: 1121-1124

[11] Holten MK, Zacho M, Gaster M, Juel C, Wojtaszewski JF, Dela F. Strength training increases insulin-mediated glucose uptake, GLUT4 content, and insulin signaling in skeletal muscle in patients with type 2 diabetes. Diabetes 2004; 53: 294-305

[12] Johnson NA, Keating SE, George J. Exercise and the liver: implications for therapy in fatty liver disorders. Semin Liver Dis 2012; 32: 65-79

[13] Keating SE, Hackett DA, George J, Johnson NA. Exercise and non-alcoholic fatty liver disease: a systematic review and meta-analysis. J Hepatol 2012; 57: 157-166
[14] Maximos M, Bril F, Portillo Sanchez P, Lomonaco R, Orsak B, Biernacki $D$, Suman A, Weber M, Cusi K. The role of liver fat and insulin resistance as determinants of plasma aminotransferase elevation in nonalcoholic fatty liver disease. Hepatology 2015; 61: 153-160

[15] Mofrad P, Contos M], Haque M, Sargeant C, Fisher RA, Luketic VA, Sterling RK, Shiffman ML, Stravitz RT, Sanyal AJ. Clinical and histologic spectrum of nonalcoholic fatty liver disease associated with normal ALT values. Hepatology 2003; 37: 1286-1292

[16] Oza N, Eguchi Y, Mizuta T, Ishibashi E, Kitajima Y, Horie H, Ushirogawa M, Tsuzura T, Nakashita S, Takahashi H, Kawaguchi Y, Oda Y, Iwakiri R, Ozaki I, Eguchi T, Ono N, Fujimoto K. A pilot trial of body weight reduction for nonalcoholic fatty liver disease with a home-based lifestyle modification intervention delivered in collaboration with interdisciplinary medical staff. J Gastroenterol 2009; 44: 1203-1208

[17] Pedersen BK, Akerström TC, Nielsen AR, Fischer CP. Role of myokines in exercise and metabolism. J Appl Physiol 2007; 103: 1093-1098

[18] Saadeh S, Younossi ZM, Remer EM, Gramlich T, Ong JP, Hurley M, Mullen KD, Cooper JN, Sheridan MJ. The utility of radiological imaging in nonalcoholic fatty liver disease. Gastroenterology 2002; 123 : 745-750

[19] Takahashi A, Abe K, Usami K, Imaizumi H, Hayashi M, Okai K, Kanno Y, Tanji N, Watanabe H, Ohira H. Simple resistance exercise helps patients with non-alcoholic fatty liver disease. Int J Sports Med 2015; 36: $848-852$

[20] Thompson D, Markovitch D, Betts JA, Mazzatti D, Turner J, Tyrrell RM. Time course of changes in inflammatory markers during a 6-mo exercise intervention in sedentary middle-aged men: a randomized-controlled trial. J Appl Physiol 2010; 108: 769-779

[21] Ueno T, Sugawara H, Sujaku K, Hashimoto O, Tsuji R, Tamaki S, Torimura T, Inuzuka S, Sata M, Tanikawa K. Therapeutic effects of restricted diet and exercise in obese patients with fatty liver. J Hepatol 1997; 27: 103-107

[22] Umpierre D, Ribeiro PA, Kramer CK, Leitão CB, Zucatti AT, Azevedo M], Gross JL, Ribeiro JP, Schaan BD. Physical activity advice only or structured exercise training and association with HbA1c levels in type 2 diabetes: a systematic review and meta-analysis. JAMA 2011; 305: 1790-1799

[23] Watanabe S, Hashimoto E, Ikejima K, Uto H, Ono M, Sumida Y, Seike M, Takei Y, Takehara T, Tokushige K, Nakajima A, Yoneda M, Saibara T, Shiota G, Sakaida I, Nakamuta M, Mizuta T, Tsubouchi H, Sugano K, Shimosegawa T. Evidence-based clinical practice guidelines for nonalcoholic fatty liver disease/nonalcoholic steatohepatitis. Hepatol Res 2015; 45: 363-377

[24] Zelber-Sagi S, Buch A, Yeshua H, Vaisman N, Webb M, Harari G, Kis O, Fliss-Isakov N, Izkhakov E, Halpern Z, Santo E, Oren R, Shibolet O. Effect of resistance training on non-alcoholic fatty-liver disease a randomized-clinical trial. World J Gastroenterol 2014; 20: 4382-4392 